\title{
Current management aspects in adult congenital heart disease: heading for the future
}

There seems to be no area of modern medicine and cardiology that has seen such great successes, as the treatment of congenital heart disease (CHD).

While 80 years ago, most children with relevant CHD died in their first years of life, nowadays more than $90 \%$ reach adulthood, often with good physical and mental health. This is predominantly the result of advances in cardiac surgery, interventional techniques, intensive care and the use of new drugs.

These achievements in the field of adults with congenital heart disease (ACHD) would not have been possible without the great commitment of leading personalities and great physicians, in particular: Joseph K. Perloff (USA), Gary Webb (Canada), Jane Somerville (UK), Carole Warnes (USA), Carlo Kallfelz, Peter Lange and John Hess (Germany). Just in time, all of them drew attention to this important and new area of cardiology and laid the foundation for optimized patient care.

The medical care of ACHD has currently reached a high, unprecedented level. However, most CHD are repaired, but not fully corrected. Therefore, anatomical and functional residua and sequelae often exist. Patients are chronically ill and require lifelong care by specialists familiar with CHD in adulthood.

Residua and sequelae, heart failure, cardiac arrhythmias, sudden cardiac death, or infective endocarditis, and, with increasing significance, acquired heart disease (e.g., coronary artery disease, valvular heart disease), pulmonary vascular disease, multi-organ complications or psychological and intellectual limitations adversely affect quality of life, performance, working ability and mental health of ACHD.

Disease progression of these chronically ill patients is influenced by factors such as genetics, environment, diet, exercise, lifestyle, stress, occupation, socioeconomic status as well as education, culture and spirituality. In addition, non-cardiac comorbidities, inadequate or false health-related information on the clinical status, and adverse health and nutritional behavior and/or wrong physical activity are distressing factors.

Therefore, despite major advances in the past, there remain still considerable challenges in the future. Still, too little attention is paid to the problems of chronic illness and there remain considerable supply deficits, especially in the areas of disease prevention, rehabilitation and holistic, innovative medical care.

ACHD who are saved from premature death with a high medical, technical and financial burden and who are generally able to lead a nearly healthy live, often lose their ability to work too early and possibly many years of life because of insufficient education and care.

To remedy these inadequately recognized deficits in ACHD care, congenital cardiologists should communicate and collaborate with specialists from other disciplines and other health professionals (such as physiotherapists, nutritionists, health economists, social workers, epidemiologists, psychologists). Prophylaxis and treatment programs should be developed for those affected to prevent, shorten or positively influence a chronic disease progression through preventive, accompanying and supportive measures.

CDTs first of two focusses issues on ACHD gives a broad review and new data to illustrate what has been achieved, but also, and with major emphasize, discusses future options and direction in the diagnosis and therapy of ACHD. We provide appropriate, state-of-the-art knowledge, about diagnosis, treatment and disease prevention in order to improve medical care and salutogenesis of ACHD.

\section{Acknowledgements}

None. 


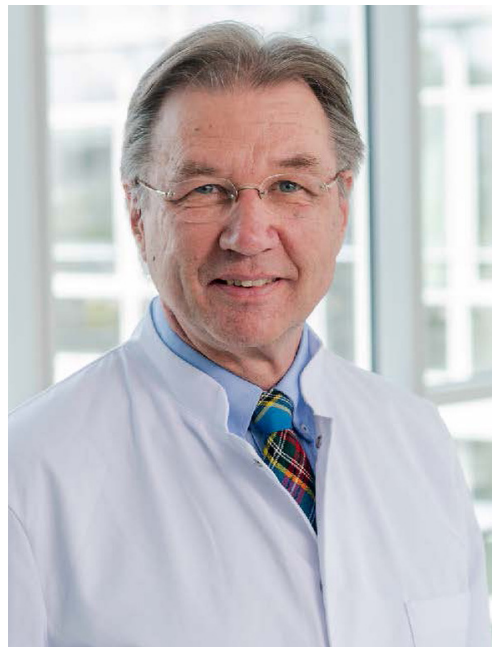

Harald Kaemmerer

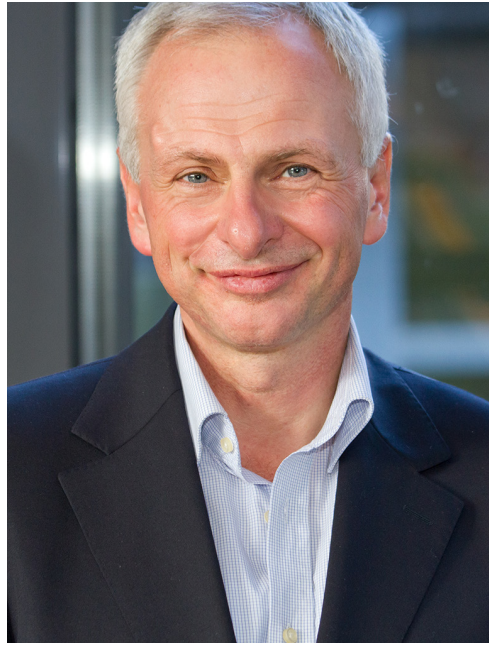

Yskert von Kodolitsch

Harald Kaemmerer, MD, VMD

Professor of Internal Medicine/Cardiology, Department of Pediatric Cardiology and Congenital Heart Disease, German Heart Center Munich, Technical University Munich, Munich, Germany.

(Email: Kaemmerer@dhm.mbn.de)

Yskert von Kodolitsch, MD, MBA

Clinic of Cardiology, University Clinic Hamburg-Eppendorf, Hamburg, Germany.

(Email:kodolitsch@uke.de)

doi: 10.21037/cdt.2018.11.09

Conflicts of Interest: The authors have no conflicts of interest to declare.

View this article at: http://dx.doi.org/10.21037/cdt.2018.11.09

Cite this article as: Kaemmerer H, von Kodolitsch Y. Current management aspects in adult congenital heart disease: heading for the future. Cardiovasc Diagn Ther 2018;8(6):696-697. doi: $10.21037 /$ cdt.2018.11.09 